\section{Aspectos críticos para a implementação da política de saúde bucal no Município de Salvador, Bahia, Brasil}

\author{
Critical issues for implementing oral health policy \\ in the city of Salvador, Bahia State, Brazil
}

\author{
${ }_{1}^{1}$ Instituto de Saúde Coletiva, \\ Universidade Federal da \\ Bahia, Salvador, Brasil. \\ Correspondência \\ C. L. M. Soares \\ Instituto de Saúde Coletiva, \\ Universidade Federal da \\ Bahia. \\ Condomínio Pedra do Sal \\ Residencias, Alameda das \\ Algas 145, Salvador, BA \\ 41620-270, Brasil. \\ catharinamatos@gmail.com
}

\begin{abstract}
This study focuses on policy implementation by public organizations. This was a qualitative, exploratory case study focusing on Brazil's National Oral Health Policy, known as "Smiling Brazil", implemented in Salvador, the State capital of Bahia. The study aimed to identify factors that facilitated or hindered the policy's implementation, based on the public policy cycle and Carlos Matus' government triangle concept, for the period from 2004 to 2007. Data collection strategies included analysis of municipal documents, interviews with key informants, and direct observation of top management meetings at the Municipal Health Secretariat. The main factors that hindered implementation of the municipal oral health policy were the project's lack of specificity; lack of human resources; and lack of financial autonomy in the Municipal Health Secretariat. The study showed that the lack of connection between the variables comprising the government triangle raised obstacles to the implementation of oral health policy in Salvador.
\end{abstract}

Oral Health; Health Policy; Public Policies
Catharina Leite Matos Soares 1 Jairnilson Silva Paim 1

\section{Introdução}

O ciclo da política pública (policy cycle) busca explicar a interação entre intenções e ações, e ao mesmo tempo relacionar o ambiente social, político e econômico de um lado e o governo do outro ${ }^{1}$. Compreende quatro momentos distintos: a determinação da agenda, a formulação da política, a implementação e a avaliação 1,2,3,4. Esses momentos não são estanques e correspondem a um processo interativo e dinâmico ${ }^{3}$.

A fase referente à implementação corresponde à etapa em que a política formulada se transforma em programa, obedecendo a diretrizes e objetivos expressos em uma legislação de caráter geral e/ou em normas regulamentadoras. Trata-se de um esforço em explicar as intenções e ações, compreendendo o momento em que os conteúdos propositivos transformam-se em ação. Nesse momento, grupos interessados agem favorecendo ou dificultando a execução das ações no interior da organização de saúde 1,2,3.

No que tange às políticas de saúde bucal, estudos revelam que no momento da implementação é importante a atuação do governo local no sucesso desta etapa, a exemplo daqueles realizados em Curitiba (Paraná), Campinas (São Paulo), Belo Horizonte e Ipatinga (Minas Gerais) e em dois municípios da Bahia 5,6,7,8.

A saúde bucal vem ganhando espaço no cenário das políticas públicas de saúde, sobretudo a partir da inserção da equipe de saúde bucal (ESB) 
na Estratégia Saúde da Família (ESF), ampliada com a formulação de política específica para a área, denominada Brasil Sorridente 9 .

A política Brasil Sorridente envolve um conjunto de ações que buscam a reorganização do serviço de saúde bucal em todos os níveis de atenção nos distintos territórios em que as ações de saúde bucal se materializam. Para tanto, recomenda a organização da atenção por meio da atenção básica, especialmente com a inclusão das ESB na ESF; estabelece a possibilidade de acesso da população a tratamentos mais complexos por meio da implantação de Centros de Especialidades Odontológicas (CEO) e Laboratórios Regionais de Prótese Dentária (LRPD); e ainda orienta a adoção de critérios de acolhimento, particularmente por linha de cuidado ou condições de vida, que podem repercutir no aumento do acesso e na qualidade da atenção da população aos serviços de saúde bucal 9 .

Estudos relacionados a essa política ratificam a sua centralidade na agenda das políticas de saúde no cenário atual 10,11,12,13, tornandose pertinente investigar como essa política vem sendo implementada nas organizações públicas de saúde. Essas organizações são o lócus onde ocorre o processo decisório, agente responsável pela implementação das políticas de saúde no âmbito do governo 14 .

Partindo dessas reflexões este estudo tem como objetivo analisar a implementação da Política Nacional de Saúde Bucal (PNSB) no Município de Salvador, Bahia, no período de 2004 a 2007, discutindo fatores que facilitaram ou dificultaram tal processo.

\section{Metodologia}

\section{Elementos teóricos}

A investigação tomou como referência o ciclo da política pública, particularmente o momento da implementação 3,4, ancorando-se no pressuposto segundo o qual "a análise da implementação de políticas de Saúde depende não apenas da correlação de forças políticas que dirigem ou pressionam o Estado e seus aparelhos, mas também da distribuição do poder no interior do aparato estatal e das formas concretas da organização do trabalho nas instituições" 15 (p. 150).

Para análise da implementação de uma política numa organização pública de saúde 16 foi utilizada a categoria governo, entendendo-a como a responsabilidade pelo comando de uma instituição, tais como um ministério, sindicato, secretaria de saúde ou unidade de saúde. Referese a três variáveis distintas: o projeto de gover- no, a governabilidade do sistema e capacidade de governo, que se entrelaçam e condicionam-se mutuamente, conformando os vértices de um triângulo, o "triângulo de governo" 17.

Para Matus 17, o projeto de governo referese ao conteúdo propositivo dos projetos de ação que um ator se propõe a realizar para alcançar seus objetivos; a governabilidade diz respeito à relação entre as variáveis que o ator controla e as que o governante não controla no seu processo de governo, variáveis estas ponderadas pelo seu valor para a ação do ator; e a capacidade de governo consiste na competência de condução ou direção. Refere-se ao acervo de técnicas, métodos, destrezas, habilidades e experiência de um ator e sua equipe de governo. Essa variável se expressa na capacidade de direção, de gerência, de administração e controle. Depende do capital intelectual acumulado pelas equipes dirigentes entendido como capital teórico, instrumental e capital experiência.

Para governar, o condutor que dirige um processo visando a alcançar seus objetivos que escolhe e altera segundo as circunstâncias (projeto de governo), precisaria superar os obstáculos de maneira não passiva, mas ativamente resistente (governabilidade do sistema) e, aliado a isto, deveria demonstrar capacidade de conduzir ou direcionar o sistema. A articulação dessas variáveis deve ocorrer de forma constante para favorecer a implementação de uma política ${ }^{17}$.

\section{Desenho do estudo}

Trata-se de um estudo exploratório, qualitativo, um estudo de caso 18 desenvolvido na Secretaria Municipal de Saúde (SMS) de Salvador no período de 2004 a 2007, tendo como referência o momento da implementação do ciclo da política pública 1,2, analisado à luz do triângulo de governo proposto por Carlos Matus 17.

Esse município, capital do Estado da Bahia, possui extensão territorial de $707 \mathrm{~km}^{2}$, população de 2.443.107 habitantes e conta com 12 distritos sanitários. Parte da coleta de dados foi realizada no distrito sanitário do subúrbio ferroviário, o mais populoso e mais antigo da cidade no que se refere à implantação de ESF.

No que concerne às ações de saúde bucal, o município apresentava, no início do estudo, um quadro de baixa cobertura de saúde bucal na ESF, cerca de $18 \%$, e mostrava ainda baixo acesso da população aos serviços e ações de saúde bucal, em torno de 6,8\%. Possuía rede insuficiente com cerca de 310 equipamentos, incluindo rede contratada e conveniada, como também oferta quase inexistente de serviços especializados em odontologia. 
As evidências foram produzidas em maio de 2007, por meio de documentos institucionais do período em estudo e também oriundos da gestão que antecedeu o período estudado, particularmente os planos de saúde e relatórios de gestão, visando a identificar as propostas e os antecedentes da política de saúde bucal, como também o Plano de Desenvolvimento Urbano do Município (PDDU). Em seguida, realizaram-se entrevistas semiestruturadas com dez dirigentes dos níveis central e distrital da SMS, quais sejam: secretário de saúde, coordenador da atenção e promoção da saúde, coordenador de recursos humanos e de saúde bucal, representante da assessoria técnica, o coordenador do distrito sanitário estudado e os gerentes de unidades de saúde tradicional, saúde da família e do CEO. As entrevistas foram gravadas e transcritas para posterior análise. De modo complementar a autora realizou observação direta de reuniões colegiadas no nível central da secretaria.

Para a definição do número de entrevistados foram identificadas na PNSB ações prioritárias a serem implantadas pelo município, dispostas em um modelo lógico entendido enquanto um "esquema visual que apresenta como um programa deve ser implementado e quais resultados são esperados" 19 (p. 46) cujos componentes, atividades e resultados derivaram do documento das diretrizes da PNSB. A identificação dessas ações orientou as escolhas dos informantes elencados com base na consulta ao organograma e regimento da SMS, envolvendo os atores que teriam alguma responsabilidade com a política em estudo, quer seja no nível central, quer seja no nível distrital.

As evidências obtidas nos documentos foram processadas em matrizes, buscando identificar relação entre o conteúdo propositivo e as ações realizadas pela gestão municipal. Adiante, extratos das entrevistas foram classificados em matriz de análise dispostos nas categorias analíticas projeto de governo, governabilidade e capacidade de governo, identificando elementos de convergência e divergência nas falas dos sujeitos envolvidos na pesquisa. Essa etapa objetivou identificar os elementos contextuais que facilitaram e/ou dificultaram a implementação da política em estudo. Extratos do diário de campo seguiram a mesma lógica de análise. Essa parte ocorreu utilizando-se a análise temática de conteúdo proposta por Minayo 20.

O projeto de investigação foi aprovado pelo Comitê de Ética em Pesquisa do Instituto de Saúde Coletiva da Universidade Federal da Bahia, com base na Resolução $n^{\circ}$. 196/96 do Conselho Nacional de Saúde.

\section{Resultados}

\section{Projeto de governo em saúde bucal na SMS}

O projeto de governo da SMS de Salvador foi centrado na organização da rede assistencial em saúde bucal, visando a: melhorar o acesso da população ao tratamento odontológico nas unidades básicas de saúde (UBS) e nas unidades de atendimento odontológico de urgência; implementar o programa Brasil Sorridente e implantar os CEO, definindo basicamente duas linhas de ação, em consonância com a política nacional Brasil Sorridente.

Merece destaque o fato de a saúde bucal constar no PDDU. O documento apresenta como diretriz a incorporação definitiva do atendimento odontológico à rede básica dos serviços de saúde do município, sugerindo que o projeto da saúde bucal iria além da SMS. Acrescenta-se que os documentos institucionais indicam a vigilância da saúde como modelo de atenção para o município, propondo o estabelecimento de diálogo com outras combinações tecnológicas, inclusive a saúde bucal.

Foi consenso entre os entrevistados que a saúde bucal fazia parte do projeto de governo na gestão que assumiu a SMS em 2005, destacandose os CEO como expressão da referida política. Houve tímida referência com relação à expansão e fortalecimento da atenção básica e também ao desenvolvimento de ações de prevenção no âmbito da SMS.

“...Vem implementando os CEO e priorizando os CEO, tendo deixado priorizado a atenção básica. É confirmada a necessidade de esses odontólogos estarem nas escolas, estarem lá fazendo a promoção da saúde, a educação na saúde..." (Entrevistado 1).

"A saúde bucal é a saúde integral. Então a gente com a implantação do CEO, que nós focamos na média complexidade (...), então não é possível imaginar um avanço na média complexidade sem estar fortalecida a atenção básica" (Entrevistado 9).

Outras ações estratégicas contidas no documento da PNSB sequer foram apontadas pelos entrevistados ou pelos documentos da instituição. No caso desses últimos, em particular o Plano Municipal de Saúde, apresentam-se sem detalhamento para a área de saúde bucal, apenas recomendando a implantação da política Brasil Sorridente.

Quanto aos componentes da PNSB que não foram contemplados pela política municipal nos documentos institucionais e nem nas falas dos entrevistados, pode-se destacar a qualificação da atenção básica por meio da incorporação de uma 
política de educação permanente para pessoal de níveis superior e médio, a utilização da epidemiologia para organização dos serviços de saúde, a implantação dos LRPD, e o estabelecimento de estratégias para a ampliação do acesso aos serviços de saúde bucal, entre outros.

Com base na análise dos documentos e entrevistas constata-se que a saúde bucal foi incorporada como proposta de governo. No entanto, as proposições apresentadas foram pouco específicas para a área, não havendo uma política municipal formulada com diretrizes e ações, demonstrando certa fragilidade no projeto (Tabela 1).

\section{Capacidade de governo na SMS}

No que concerne à capacidade de governo, observou-se que a partir de 2005 o gestor municipal da saúde apresentava formação em saúde coletiva, com conhecimento sobre o campo e também acerca da PNSB e que a equipe de coordenação possuía profissionais diversificados, dentre os quais alguns com conhecimento em saúde coletiva e experiência em gestão, e outros não, compondo uma equipe heterogênea.

As evidências indicam que a SMS apresentava dificuldades em acompanhar as políticas federais, requerendo do município estruturação da rede de serviços de saúde bucal para tanto. Nesse caso, o município buscava estratégias que possibilitassem a implantação do programa Brasil Sorridente, objetivando o aproveitamento da oportunidade disponibilizada pelo governo federal, no sentido de assegurar recursos.
"A gente não tem uma agilidade suficiente para estar acompanhando as estratégias que o ministério trilha, não é? (...) pra você achar na rede só especialistas, e tal, quer dizer, Brasília solta a solução, mas muitas vezes cria um problema pra gente. Então para correr atrás da oportunidade, a gente tem que adequar, tem que fazer, mas aqui, tem-se feito um esforço" (Entrevistado 1).

“Toda inclusão do PSF [Programa Saúde da Família] é feita via parceiros. (...) Nós não temos na rede uma história de concursos feitos para especialista. Para você encontrar um especialista, muitas vezes pelo valor que a prefeitura paga, você não encontra" (Entrevistado 1).

A SMS tem optado pela terceirização da mão de obra, inserindo todo pessoal via empresas contratadas, utilizando, portanto, funcionários precarizados. Ressalta-se a não existência de profissionais de odontologia na reserva técnica da prefeitura, pela ausência de concurso contemplando esta categoria nos últimos anos. Acrescenta-se a não realização de concursos para especialistas no interior da rede de serviços de saúde do município, considerando a implantação dos CEO e os baixos salários oferecidos pela instituição não atrair tais profissionais, dificultando a contratação para a área, apesar de a contratação de especialistas não ser prerrogativa para a implantação dos CEO.

Há ainda a inexistência de plano de cargos, salários e carreiras para os profissionais de saúde da Prefeitura Municipal de Salvador. Devido às baixas remunerações, os profissionais da rede apresentavam-se descompromissados com o projeto de governo e buscam outras atividades

Tabela 1

Síntese das propostas de governo apresentadas pela Secretaria Municipal de Saúde (SMS) de Salvador, Bahia, Brasil.

\begin{tabular}{|c|c|}
\hline Período & Propostas \\
\hline \multirow[t]{3}{*}{$2002-2005$} & - Ampliação das ações de odontologia sanitária para as equipes de PSF \\
\hline & - Redução da incidência de doenças bucais de 5 a 14 anos \\
\hline & - Contratação de odontólogos para a rede SMS \\
\hline \multirow[t]{6}{*}{ 2006-2009 } & $\begin{array}{l}\text { - Incorporação definitiva do atendimento odontológico à rede básica dos serviços de saúde do } \\
\text { município }\end{array}$ \\
\hline & - Eleição da vigilância da saúde como modelo de atenção para o município \\
\hline & - Organização da rede assistencial em saúde bucal \\
\hline & - Melhoria do acesso da população aos serviços de saúde bucal nas UBS e nas UAO \\
\hline & $\begin{array}{l}\text { - Implementação do programa Brasil Sorridente (ampliação da atenção básica com a implantação da } \\
\text { ESB na ESF) }\end{array}$ \\
\hline & - Implementação dos CEO \\
\hline
\end{tabular}

CEO: Centros de Especialidades Odontológicas; ESB: equipe de saúde bucal; ESF: Estratégia Saúde da Família;

PSF: Programa Saúde da Família; UAO: unidade básica tradicional; UBS: unidade básica de saúde. 
para complementar o seu salário, gerando duplos vínculos dos profissionais e, impossibilitando-os assim de cumprir a carga horária.

Em síntese, a SMS de Salvador apresenta baixa capacidade de governo decorrente da fragilidade da política de gestão do trabalho no município, associada à Lei Orgânica da Prefeitura, que não permite a contratação de pessoal por tempo determinado, e também à Lei de Responsabilidade Fiscal, que limita os gastos com contratação de pessoal.

“...A dificuldade maior do engessamento da contratação, isso vem todo por conta da lei de responsabilidade fiscal, da atual lei orgânica da prefeitura, que ao meu ver tinha que estar sendo mexida, eu até conversei com um vereador sobre isso, porque na maioria dos municípios você tem contrato por tempo determinado para alguma ação, e aqui você não tem. Quer dizer, a prefeitura fica engessada, ai tem que contratar serviço, que é mais caro, e como você quer crescer a rede sem contratar serviço?" (Entrevistado 1).

Apesar de o secretário de saúde e parte da sua equipe terem formação em saúde coletiva e experiência de gestão, problemas da área de recursos humanos constituíram-se no principal obstáculo no que concerne à capacidade de governo durante a implementação da Política $\mathrm{Mu}$ nicipal de Saúde Bucal. Nesse particular pode-se destacar: inexistência de planos de cargos e salários, múltiplas formas de inserção dos profissionais de saúde bucal (contratos temporários, concurso público e terceirizações) e precarização dos vínculos dos profissionais da ESF. Além disso, a insuficiência de dentistas na rede e os limites de gasto com salários decorrentes da Lei de Responsabilidade Fiscal constrangeram a oferta de serviços para a população.

\section{Governabilidade na SMS}

No período estudado, a governabilidade na secretaria foi comprometida tanto no âmbito externo, refletindo o diálogo do secretário de saúde com o gestor do executivo municipal, quanto no plano interno, tratando-se da relação com a equipe de dirigentes e técnicos da referida secretaria, dentre outros atores do processo de governo.

Houve pouca adesão do prefeito aos projetos apresentados pelo secretário de saúde, apesar da utilização dos projetos da saúde como slogan para promover a sua gestão. Acrescenta-se o fato de o orçamento ter sido elaborado de acordo com a Emenda Constitucional $n^{\circ}$. 29, ou seja, nos $15 \%$, e relatos mostram que este recurso não foi efetivamente disponibilizado para a Secretaria de Saúde, reduzindo o grau de liberdade da SMS para decisão e utilização do recurso.
“...Entramos 2007 com 60 milhões de restos a pagar, ou seja, de um orçamento de 160 que nós tivemos em 2006, 100 foram pagos e 60 não foram pagos. Então isso demonstra que na prática, efetivamente, houve outras prioridades na prefeitura que não foram a saúde. Como é que deixa sem pagar 60 milhões? Isso desestrutura qualquer tipo de serviço. E olha que esses 160 milhões correspondiam, ao mínimo, aos 15\%...” (Entrevistado 7).

“...Então quando você vê, por exemplo, hoje saiu no jornal o prefeito dizendo aos prestadores de serviço de saúde, de ortopedia, que ia devolver os $25 \%$ que foram cortados, cortados por quê? Porque era superfaturamento, porque era fraude, era roubo, não sei que, dizendo que você vai devolver? Você tá botando fim a essa gestão da Secretaria de Saúde, não dá pro mesmo grupo continuar, porque esse gestor municipal é o principal opositor por exemplo, e agora se aproximando das eleições municipais no ano que vem ele quer se reeleger, é o principal opositor de uma proposta" (Entrevistado 8).

A falta de autonomia financeira sobre os recursos destinados à saúde e a falta da gerência destes recursos foram o ponto crítico desse processo, pois deixou margem para que outro ator no processo de governo, a Secretaria da Fazenda, definisse as prioridades de pagamento. Nesse particular impediu que a Secretaria de Saúde negociasse seus contratos e pagasse seus fornecedores; atrasou o pagamento ou pagou parcialmente os prestadores de serviços, fornecedores de material ou empresas de manutenção; comprometeu a sustentabilidade das ações de saúde bucal, já que o processo de trabalho em odontologia é dependente de tecnologias materiais, equipamentos e insumos.

"Quem administra a verba ainda é o tesouro municipal. Ao meu ver isso toma da gente a liberdade de estar arbitrando essa utilização. Porque se nós tivéssemos esses 15\% conosco, você tem o todo e diz: vou priorizar isso, isso e isso. Muitas vezes a prioridade lá do palácio municipal não é a nossa aqui. (...) a gente fez a vontade do gestor e basta que o fornecedor não receba da fonte da prefeitura para que ele tenha medo de fornecer no próximo mês" (Entrevistado 1).

O ano de 2006 foi um período de instabilidade política para a gestão municipal devido à crise financeira sofrida pela prefeitura, que acarretou repasses atrasados dos recursos para o pagamento dos profissionais das ESF, gerando constantes manifestações e paralisações dos serviços de saúde prestados por eles.

Somam-se a essa instabilidade política, divergências ideológicas acerca dos projetos de saúde bucal no interior da SMS. Por um lado, existiam profissionais da ESF que colocavam 
seus interesses particulares em detrimento da construção do Sistema Único de Saúde (SUS); por outro, observava-se os profissionais da rede municipal, que ingressaram via concurso público e que demonstram maior compromisso na construção do sistema, exigindo maior perícia da coordenação de saúde bucal para mediar a multiplicidade de interesses.

A linha estratégica da atenção básica, relacionada à expansão das ESB no PSF, mostrou-se bastante comprometida face aos problemas financeiros apresentados. Em que pese o apoio do Ministério da Saúde favorecendo a implantação das ESF, a contrapartida da prefeitura no que diz respeito a manter o serviço em funcionamento não ocorreu, comprometendo a política de saúde bucal no município.

No nível distrital, semelhante ao nível central, a pouca autonomia financeira gerava dependência do nível central. O distrito sanitário recebia um adiantamento no valor de $\mathrm{R} \$ \mathbf{8 0 0 , 0 0}$ (oitocentos Reais) para conduzir toda a política de saúde no nível distrital, sendo incompatível com as necessidades do distrito.

A análise sugere que o secretário de saúde apresentou pouca governabilidade para implementar as políticas de saúde bucal no município, tanto no âmbito externo quanto no interno (Tabela 2).

\section{Discussão}

Os resultados deste estudo evidenciaram que houve pouca articulação do triângulo de governo, não favorecendo a implementação da polí- tica de saúde bucal no Município de Salvador. Das questões apresentadas destacam-se a ausência de autonomia financeira da SMS, a falta de comprometimento do prefeito com a política e também a fragilidade da política de gestão de trabalho no município.

O projeto de governo em saúde bucal apresentou-se frágil e inespecífico. Vale ressaltar que o plano do município foi formulado de forma ascendente, democrática. Com referência à saúde bucal, embora seus problemas tenham sido mencionados nos 12 distritos sanitários do município, o grupo técnico não obteve um nível de debate e acúmulo de poder que assegurasse a inclusão de suas ações no Plano Municipal de Saúde, reforçando a hipótese de que a área não possuía um projeto claramente definido a ser empreendido.

Contrariamente, o estudo de Lopes 10 aponta a importância de conceber um projeto prévio para facilitar o processo de implementação de políticas ratificado por Bermudez 21, afirmando que todas as instituições deveriam seguir políticas escritas, que incluiriam as diretrizes e as responsabilidades dos implementadores, pois a ausência de um projeto explicitado produz dificuldades não apenas no campo da análise política como internamente para a reprodução na ponta do sistema.

Garcia 13 sustenta tal argumento no seu estudo acerca da elaboração do programa Brasil Sorridente, afirmando que por ter concebido um projeto prévio facilitou o processo da implementação. Dessa forma, a saúde bucal foi incorporada na agenda do executivo-presidente, desenhou o perfil desejável para o ministro da

Tabela 2

Síntese dos principais fatos referentes à governabilidade no âmbito da Secretaria Municipal de Saúde (SMS) de Salvador, Bahia, Brasil.

Governabilidade no âmbito da SMS

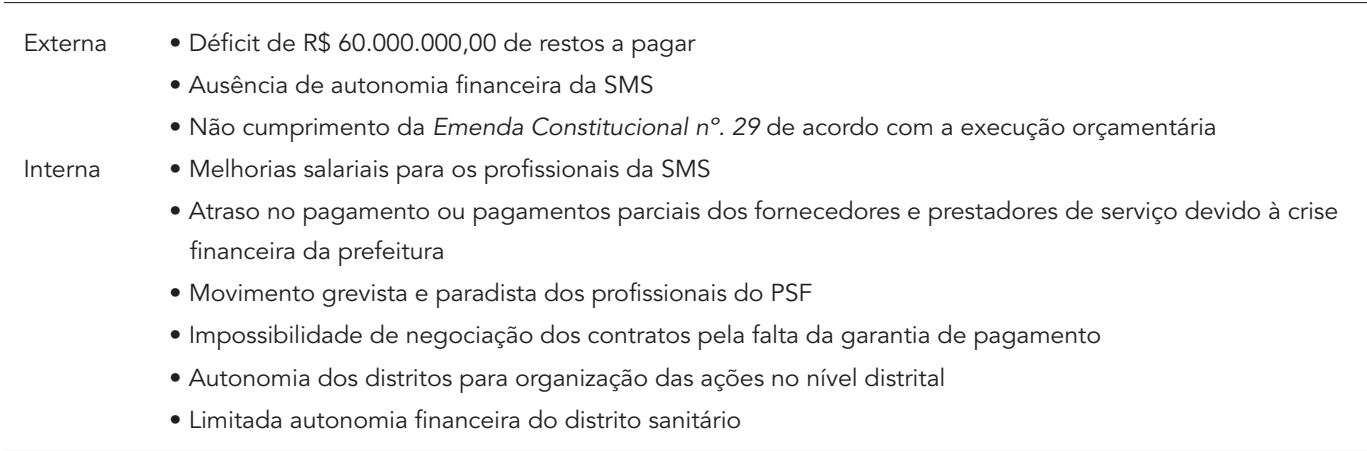

PSF: Programa Saúde da Família 
saúde e conseguiu transformar a Área Técnica de Saúde Bucal em Coordenação Nacional de Saúde Bucal.

O presente estudo revelou um contexto de baixa governabilidade, atribuído dentre outros fatores à ausência de autonomia financeira da SMS. A falta de gerência dos recursos provenientes do tesouro municipal foi responsável por desencadear muitos outros processos que, direta ou indiretamente, mantinham relação com a saúde bucal. Impediu o secretário de negociar os contratos, pagar os fornecedores e empresas de manutenção, garantir o pagamento do pessoal inserido via empresa terceirizada, e manter a regularidade no fornecimento de insumos.

Achados semelhantes acerca da falta de autonomia financeira foram encontrados nos estudos de Lopes 10 e Vilasbôas \& Paim ${ }^{22}$. O primeiro refere que o apoio do executivo-prefeito viabilizou a implementação dos programas Dentescola e Carioca Rindo à Toa no Rio de Janeiro, e o segundo, a perícia do dirigente da SMS criou viabilidade política suficiente para o desenvolvimento do SUS municipal.

A governabilidade do sistema depende, dentre outros fatores, do capital político do dirigente, ou seja, seu poder ou peso político. A análise do Município de Salvador revela o baixo peso político que a saúde e seus projetos representaram para a prefeitura da cidade. Cabe esclarecer que o período estudado compreendeu um governo formado pela coligação de vários partidos, com distintas bases políticas e ideológicas, e que coube ao Partido dos Trabalhadores (PT) a SMS nessa conformação, representando a minoria na composição geral das secretarias de governo.

No caso em estudo, o executivo prefeito revelou-se um opositor dos projetos da SMS, dificultando a implementação da PNSB. Kerbauy 23 destaca que o executivo-prefeito se constitui em agente de maior peso no processo de implementação de políticas, tendo as secretarias municipais papel secundário. Elucida que essa tendência respalda-se na Lei de Responsabilidade Fiscal e seus efeitos, que estabelecem punições penais como reclusão e multas, favorecendo a centralização do poder decisório na figura do prefeito.

As questões relativas aos recursos humanos foram entraves à política de saúde bucal. O reduzido número de profissionais, a ausência de qualificação, bem como as várias formas de inserção deles na SMS, criaram um cenário heterogêneo para os profissionais de saúde bucal do município, apresentando múltiplos projetos em disputa, que dificultaram o processo de implementação da política, considerando que exige do gestor da SMS maior perícia para formar um projeto comum. Estudos sobre implementação de políticas em âmbito municipal confirmam tal argumento, nos quais experiências consideradas exitosas na área de saúde bucal nos municípios do Rio de Janeiro 10 e Curitiba 5 demonstraram que foi necessário aumentar o número de profissionais, ao mesmo tempo em que os capacitava para empreender novos projetos.

Sinteticamente pode-se afirmar que a SMS de Salvador apresentou um contexto de pouca governabilidade do secretário de saúde e sua equipe para empreender os projetos da saúde, baixa capacidade de governo, e também ausência de um projeto de saúde bucal consistente, que resultaram em barreiras para a implementação da política em estudo no município, não havendo articulação das variáveis que compõem o triângulo de governo. De modo distinto, os estudos de Chaves \& Silva 8 e Vilasbôas \& Paim ${ }^{22}$, realizados na Bahia, apontaram que a articulação dos elementos que fazem parte do triângulo de governo foi facilitadora no processo de implementação de projeto de reorganização de práticas de saúde bucal em dois municípios baianos, bem como na implementação da política de saúde, respectivamente. A investigação sugere que o apoio do poder local parece ser imprescindível no momento da implementação da política, o que não ocorreu no Município de Salvador no período de estudo.

\section{Considerações finais}

Os resultados desta pesquisa reforçam a tese de que o governo local é considerado um agente importante no processo de implementação de uma política de saúde. Nesse sentido, a falta de apoio do executivo prefeito foi um obstáculo significativo à Política Municipal de Saúde Bucal de Salvador.

No caso particular, a ausência de autonomia financeira da SMS muito contribuiu para o insucesso, pois desencadeou uma série de outros problemas para a política em estudo, tais como: falta de regularidade no fornecimento de insumos para as unidades de saúde e na manutenção dos equipamentos odontológicos; falta de pagamento ao pessoal terceirizado lotado nas unidades de saúde da família e nos CEO, inviabilizando o desenvolvimento do trabalho nestas unidades. Convém ressaltar que o processo de trabalho em saúde bucal apresenta certa dependência das tecnologias materiais como meios de trabalho 24 .

A falta de articulação das variáveis que compõem o triângulo de governo foi um ponto chave no que se refere à política em estudo. A fragilidade do projeto, aliada à baixa capacidade de governo e à reduzida governabilidade foram 
entraves à implementação da política de saúde bucal no Município de Salvador.

Os recursos humanos configuraram um obstáculo a mais para a política em questão, pois a ausência de profissionais impossibilitou a ex- pansão da rede e dos serviços de saúde bucal. Por outro lado, as múltiplas formas de inserção dos profissionais dentistas impuseram à gestão da SMS restrições na construção de um projeto de saúde bucal comum.

\section{Resumo}

O estudo consiste em investigação acerca da implementação de políticas em organizações públicas. Trata-se de uma pesquisa qualitativa, um estudo de caso, exploratório, cujo objeto foi a Política Nacional de Saúde Bucal, Brasil Sorridente, no Município de Salvador, Bahia, Brasil. Buscou-se identificar fatores que facilitaram ou dificultaram a implementação da referida política, com base no ciclo da política pública e à luz do triângulo de governo, no período de 2004 a 2007. Como estratégias de coleta de dados foram consultados documentos municipais, feitas entrevistas com informantes-chave e observação direta em reuniões do nível central da Secretaria Municipal de Saúde (SMS). Os resultados apontaram que os principais fatores que dificultaram a implementação da política municipal de saúde bucal foram: a inespecificidade do projeto; os recursos humanos; e a falta de autonomia financeira da SMS. O estudo revelou que a desarticulação entre as variáveis que compõem o triângulo de governo representou obstáculos à implementação da política de saúde bucal no município estudado.

Saúde Bucal: Política de Saúde; Políticas Públicas

\section{Colaboradores}

C. L. M. Soares desenvolveu a pesquisa e elaborou o texto do artigo. J. S. Paim orientou o trabalho e revisou o artigo. 


\section{Referências}

1. Kelly RM, Palumbo D. Theories of policy making. In: Hawkesworth M, Kogan M, editors. Encyclopedia of government and politics. London: Routledge; 1992. p. 644-55.

2. Viana AL. Abordagens metodológicas em políticas públicas. Rev Adm Pública 1996; 30:5-43.

3. Pinto ICM. Ascensão e queda de uma questão na agenda governamental: o caso das organizações sociais da saúde na Bahia [Tese de Doutorado]. Salvador: Escola de Administração. Universidade Federal da Bahia; 2004.

4. Cunha EM. Regra e realidade na constituição do SUS municipal: implementação da NOB $96 \mathrm{em}$ Duque de Caxias [Dissertação de Mestrado]. Rio de Janeiro: Escola Nacional de Saúde Pública; Fundação Oswaldo Cruz; 2001.

5. Serra CG. A saúde bucal como política de saúde, análise de três experiências recentes: Niterói, Campinas e Curitiba [Dissertação de Mestrado]. Rio de Janeiro: Instituto de Medicina Social, Universidade do Estado do Rio de Janeiro; 1998.

6. Roncalli AG. A organização da demanda em serviços públicos de saúde bucal: universalidade, eqüidade e integralidade em saúde bucal coletiva [Tese de Doutorado]. Araçatuba: Faculdade de Odontologia, Universidade Estadual Paulista Júlio de Mesquita Filho; 2000.

7. Peres MAA. A saúde bucal em um sistema local de saúde: estudo de caso no Município de Ipatinga, MG, Brasil, 1989-1994 [Dissertação de Mestrado]. São Paulo: Faculdade de Saúde Pública, Universidade de São Paulo; 1995.

8. Chaves SCL, Vieira-da-Silva LM. Atenção à saúde bucal e a descentralização da saúde no Brasil: estudo de dois casos exemplares no Estado da Bahia. Cad Saúde Pública 2007; 23:1119-31.

9. Ministério da Saúde. Diretrizes da Política Nacional de Saúde Bucal. Brasília: Ministério da Saúde; 2004.

10. Lopes MGM. Política "Carioca Rindo à Toa" e o Programa Saúde e Cidadania, "Dentescola”: análise da política de saúde bucal no Município do Rio de Janeiro e sua trajetória rumo à implementação considerando a interface com o "Brasil Sorridente" [Dissertação de Mestrado]. Rio de Janeiro: Instituto de Medicina Social, Universidade do Estado do Rio de Janeiro; 2007.

11. Guerra KCM. Os Centros de Especialidades Odontológicas nos municípios do Estado do Rio de Janeiro: uma investigação dos fatores identificáveis como facilitadores ou não na implantação de uma política de indução financeira [Dissertação de Mestrado]. Rio de Janeiro: Instituto de Medicina Social, Universidade do Estado do Rio de Janeiro; 2009.

12. Bartole MCS. Da boca cheia de dentes ao Brasil Sorridente: uma análise retórica da formulação da Política Nacional de Saúde Bucal [Dissertação de Mestrado]. Rio de Janeiro: Instituto de Medicina Social, Universidade do Estado do Rio de Janeiro; 2006.
13. Garcia DV. A construção da política nacional de saúde bucal: percorrendo os bastidores do processo de formulação [Dissertação de Mestrado]. Rio de Janeiro: Instituto de Medicina Social, Universidade do Estado do Rio de Janeiro; 2006.

14. Grupo de Análise de Políticas de Inovação. Metodologia de análise de políticas públicas. São Paulo: Universidade Estadual de Campinas; 2002.

15. Paim JS. Aspectos críticos da institucionalização da planificação em organizações públicas de saúde. In: Paim JS, organizador. Saúde: política e reforma sanitária. Salvador: Instituto de Saúde Coletiva; 2002. p. 407-43.

16. Paim JS. Burocracia e aparato estatal: implicações para a planificação e implementação de políticas de saúde. In: Paim JS, organizador. Saúde: política e reforma sanitária. Salvador: Instituto de Saúde Coletiva; 2002. p. 149-62.

17. Matus C. Política, planejamento e governo. 2a Ed. Brasília: Instituto de Pesquisa Econômica Aplicada; 1996.

18. Yin RK. Estudo de caso: planejamento e métodos. Porto Alegre: Bookman; 2005.

19. Hart Z, Silva LMV, Chaves SCL, Silva GA. Uso de modelos teóricos na avaliação em saúde: aspectos conceituais e operacionais In: Hart Z, Vieira-da-Silva LM, organizadoras. Rio de Janeiro: Editora Fiocruz/ Salvador: EdUFBA; 2005. p. 41-64.

20. Minayo MCS. O desafio do conhecimento: pesquisa qualitativa em saúde. São Paulo: Editora Hucitec/Rio de Janeiro: ABRASCO; 2006.

21. Bermudez JAZ. Formulação, implementação e componentes de uma política de medicamentos. In: Seminário Internacional sobre Acesso a Medicamentos: Direito Fundamental e Papel do Estado. http://www.opas.org.br/medicamentos/seminar/ acesso/apresent/bermude1.pdf (acessado em 15/ Ago/2006).

22. Vilasbôas ALQ, Paim JS. Práticas de planejamento e implementação de políticas no âmbito municipal. Cad Saúde Pública 2008; 24:1239-50.

23. Kerbauy MTM. Descentralização, formulação e implementação de políticas públicas. In: Anais do 3o Encontro da Associação Brasileira de Ciência Política. Rio de Janeiro: Associação Brasileira de Ciência Política; 2002. p. 17.

24. Mendes-Gonçalves RB. Tecnologia e organização social das práticas de saúde: características tecnológicas de processo de trabalho na rede estadual de centros de saúde de São Paulo. São Paulo: Editora Hucitec; 1994.

Recebido em 04/Ago/2010

Versão final reapresentada em 16/Mar/2011 Aprovado em 29/Mar/2011 